\title{
A REMARK ON THE GROWTH OF SOLUTIONS OF FIRST ORDER ALGEBRAIC DIFFERENTIAL EQUATIONS
}

\author{
SH. STRELITZ
}

AbSTRaCr. Two theorems on the growth of complex- and real-valued solutions of first order algebraic differential equations are proven.

1. Consider the algebraic differential equation

$$
y^{\prime 2}=a y^{3}+b y^{2}+c y+d
$$

with certain complex constants $a, b, c, d$. It is known (see [3] and other references cited there) that given any comparison function this equation has complex-valued solutions whose absolute values grow more rapidly than the comparison function. In other words, there is no majorant expressed in terms of the coefficients of the equation for the solution of the differential equation on the $x$ axis.

We consider the equation

$$
F\left(x, y, y^{\prime}\right)=0
$$

with real $x$ and complex-valued (or real-valued) $y$, where $F$ is a polynomial in respect to $y$ and $y^{\prime}$. Some estimates of the growth of solutions under corresponding conditions imposed on (1) were found in [1], [2].

We show below that for equations from a certain large subset $E$ of equations of type (1) a majorant for their solutions on the $x$ axis can be built.

2. In order to formulate the conditions defining the set $E$ we rewrite (1) in the following way:

$$
F_{0}\left(x, y, \frac{y^{\prime}}{y}\right) \equiv \sum_{j=0}^{n} P_{j}\left(x, \frac{y^{\prime}}{y}\right) y^{n-j}=0
$$

with

$$
P_{j}(x, u)=\sum_{k_{j}=0}^{m_{j}} F_{j k_{j}}(x) u^{m_{j}-k_{j}}, \quad j=0,1,2,3, \ldots, n .
$$

Definition. A differential equation (2) with complex-valued coefficients $F_{m l}(x)$ belongs to $E$ if and only if:

(1) $m_{0} \geqslant m_{j} ; j=1,2, \ldots, n$ (see (3)),

(2) $\left|F_{00}(x)\right|>0, x \in[0, \infty)$, and

(3) $F_{k j}(x)$ are continuous functions on $[0, \infty)$ for all possible $k$ and $j$.

Received by the editors March 8, 1979.

AMS (MOS) subject classifications (1970). Primary $34 \mathrm{Cl} 10$.

Key words and phrases. Algebraic differential equations, growth of solutions.

(C) 1980 American Mathematical Society 0002-9939/80/0000-0161/\$02.25 
THEOREM 1. Let $y(x)$ be a differentiable complex-valued solution of the differential equation (2) which belongs to $E$ on the ray $\left[x_{0}, \infty\right)$. Then

$$
y(x)=O\left(\exp \int_{x_{0}}^{x} V(t) d t\right)
$$

where

$$
V(x)=\max _{\substack{0<k_{j}<m_{j} \\ j=0,1,2, \ldots, n}}\left\{\left|\frac{F_{j k_{j}}(x)}{F_{00}(x)}\right|^{1 / k_{j}}, 1\right\}
$$

We will also prove:

THEOREM 2. Let

$$
F\left(x, y, y^{\prime}\right) \equiv \sum_{j=0}^{n} y^{n-j} \sum_{k_{j}=0}^{m_{j}} F_{j k_{j}}(x) y^{\prime m_{j}-k_{j}}=0
$$

be given with continuous functions $F_{j k_{j}}(x)$ on $[0, \infty)$ and

$$
\begin{aligned}
\left|F_{00}(x)\right| \geqslant a_{0}>0, \quad\left|F_{0 m_{0}}(x)\right| \geqslant a_{0}>0, \quad a_{0}=\text { Const, } & \\
& m_{0} \geqslant m_{j} ; j=1,2, \ldots, n .
\end{aligned}
$$

Then for each differentiable real-valued solution $y(x)$ on the ray $\left[x_{0}, \infty\right)$ one has

$$
y(x)=O\left(V_{0}(x)\right)
$$

where

$$
V_{0}(x)=\operatorname{Sup}_{\substack{0<k_{j}<n_{j} \\ 0<j<n}}\left|F_{j k_{j}}(x)\right|
$$

We will prove both of these theorems in the following sections of this paper.

3. Proof of Theorem 1. Because of the condition (2) in the definition of the set $E$ we can put $F_{00}(x) \equiv 1$. Let $y(x)$ be a solution of (2) defined on $\left[x_{0}, \infty\right)$. We replace $x$ by $t$ according to the equality

$$
t=\int_{x_{0}}^{x} V(x) d x
$$

where $V(x)$ is defined in (5). Then $d y / d x=V(x) d y / d t$ and

$$
F_{0}\left(x, y, \frac{y^{\prime}}{y}\right)=\sum_{j=0}^{n} y^{n-j} \sum_{k_{j}=0}^{m_{j}} F_{j k_{j}}(x) V^{m_{j}-k_{j}}(x)\left(\frac{y_{t}^{\prime}}{y}\right)^{m_{j}-k_{j}}=0 \text {. }
$$

Dividing the last equation by $V^{m_{0}}(x) y^{n}$ we obtain

$$
\sum_{k=0}^{m_{0}} F_{0 k_{0}}(x) V^{-k_{0}}(x)\left(\frac{y_{t}^{\prime}}{y}\right)^{n-k_{0}}=-\sum_{j=0}^{n-1} y^{-j} \sum_{k_{j}=0}^{m_{j}} F_{j k_{j}}(x) V^{m_{j}-m_{0}-k_{j}}(x)\left(\frac{y_{t}^{\prime}}{y}\right)^{m_{j}-k_{j}} \text {. }
$$


Note now that

$$
\frac{\left|F_{0 k_{0}}(x)\right|}{V^{k_{0}}(x)}=\left(\sqrt[k_{0}]{\frac{F_{0 k_{0}}(x) \mid}{V(x)}}\right)^{k_{0}}<1
$$

and

$$
\frac{\left|F_{j k_{j}}(x)\right|}{V^{m_{0}-m_{j}+k_{j}(x)}} \leqslant \frac{\left|F_{j k_{j}}(x)\right|}{V^{k_{j}}(x)}<1 .
$$

Thus the coefficients of (2.3) are bounded by 1 .

4. Case 1. Suppose first that there is an infinite sequence of points $t_{p} \uparrow \infty$ such that:

(a) $t_{p}$ is a local maximal point of the function

$$
\varphi(t)=\ln |y(t)| / t \text { and }
$$

(b)

$$
\varlimsup_{t \rightarrow \infty} \varphi(t)=\lim _{p \rightarrow \infty} \varphi\left(t_{p}\right)=\infty
$$

(if $\varlimsup_{t \rightarrow \infty} \varphi(t)<\infty$ then Theorem 1 is correct).

Under these assumptions

$$
\varphi^{\prime}\left(t_{p}\right)=\frac{\left|y\left(t_{p}\right)\right|^{\prime}}{\left|y\left(t_{p}\right)\right|} \cdot \frac{1}{t_{p}}-\frac{\ln \left|y\left(t_{p}\right)\right|}{t_{p}^{2}}=0 \Rightarrow \frac{\left|y\left(t_{p}\right)\right|^{\prime}}{\left|y\left(t_{p}\right)\right|}=\frac{\ln \left|y\left(t_{p}\right)\right|}{t_{p}}=\varphi\left(t_{p}\right)
$$

and

$$
\left|\frac{y^{\prime}\left(t_{p}\right)}{y\left(t_{p}\right)}\right| \geqslant \frac{\left|y\left(t_{p}\right)\right|^{\prime}}{\left|y\left(t_{p}\right)\right|}=\varphi\left(t_{p}\right) \underset{p \rightarrow \infty}{\rightarrow} \infty
$$

Since $\left|y^{\prime} / y\right|>1$ at $t_{p}$, it then follows from (11) and (17) that

$$
\left|\frac{y^{\prime}}{y}\right|^{m_{0}}-\sum_{j=1}^{m_{0}}\left|\frac{y^{\prime}}{y}\right|^{m-j}<\left|\frac{y^{\prime}}{y}\right|^{n} \sum_{j=1}^{n} \frac{1}{|y|^{j}}-\frac{n}{|y|}\left|\frac{y^{\prime}}{y}\right|^{n}
$$

and since $\left|y\left(t_{p}\right)\right| \underset{p \rightarrow \infty}{\rightarrow} \infty$ one has $(1+o(1))\left|y^{\prime} / y\right|^{m_{0}}<0$. The last inequality is impossible. Hence in (15) one must have

$$
\varlimsup_{p \rightarrow \infty} \varphi\left(t_{p}\right)<C<\infty \Rightarrow \varlimsup_{t \rightarrow \infty} \varphi(t)=\varlimsup_{t \rightarrow \infty} \frac{\ln |y(t)|}{t}<C
$$

where $C$ is a certain constant dependent on the solution $y(x)$ and satisfies

$$
\ln |y(t)|<C^{\prime} t \Rightarrow \ln |y(x)|<C \int_{x_{0}}^{x} V(x) d x,
$$

which completes the proof of Theorem 1 for Case 1 .

Case 2. $\varphi(t) \uparrow \infty, t \geqslant t_{0}, t \rightarrow \infty$. In this case one has

$$
\varphi^{\prime}(t)=\frac{1}{t}\left\{\frac{|y(t)|^{\prime}}{|y(t)|}-\frac{\ln |y(t)|}{t}\right\} \geqslant 0 \Rightarrow \frac{\ln |y(t)|}{t}<\frac{|y(t)|^{\prime}}{|y(t)|}<\left|\frac{y^{\prime}(t)}{y(t)}\right|
$$


so that $\left|y^{\prime}(t) / y(t)\right| \underset{t \rightarrow \infty}{\rightarrow} \infty$. Repeating the same considerations as in the last paragraph we conclude that $\varphi(t)<C$ which completes the proof of Theorem 1.

5. Proof of Theorem 2. The proof of this theorem is similar in its method to the proof of Theorem 1. We substitute now $x$ for $t$ from $t=\int_{x_{0}}^{x} V_{0}(x) d x$ with

$$
V_{0}(x)=\operatorname{Sup}_{\substack{0<k_{j}<m_{j} \\ 0<j<n}}\left|F_{j k_{j}}(x)\right| .
$$

We assume $F_{00}(x) \equiv 1$ and get:

$$
\sum_{j=0}^{n} \sum_{k_{j}=0}^{m_{j}} \frac{F_{j k_{j}}(x)}{V_{0}^{-m_{j}+m_{0}+k_{j}}(x)} y^{\prime m_{j}-k_{j y}} y^{j}=0
$$

where, for all $j$ and $k_{j}$,

$$
\left|\frac{F_{j k_{j}}(x)}{V_{0}^{m_{0}-m_{j}+k_{j}(x)}}\right| \leqslant 1
$$

(a) Suppose there is a sequence $t_{p} \uparrow \infty$ such that, for each $p,\left|y\left(t_{p}\right)\right|$ is a local maximal value and

$$
\lim _{p \rightarrow \infty}\left|y\left(t_{p}\right)\right|=\varlimsup_{t \rightarrow \infty}|y(t)|
$$

Then $y^{\prime}\left(t_{p}\right)=0$ and from (18) and (19) it follows that

$$
\left|y\left(t_{p}\right)\right|^{n} \leqslant C \sum_{k=0}^{n-1}\left|y^{k}\left(t_{p}\right)\right|
$$

with a certain constant $C>0$. If $\left|y\left(t_{p}\right)\right| \leqslant 1$ then $|y(x)|=O(1)$. Suppose instead that $\left|y\left(t_{p}\right)\right| \uparrow \infty$. Then for $p$ large enough $\left|y\left(t_{p}\right)\right|^{n}<C n\left|y\left(t_{p}\right)\right|^{n-1}$ and $(1+o(1))\left|y\left(t_{p}\right)\right|^{n} \leqslant 0$. The last inequality is impossible. Thus in case (a) one has $y(x)=O(1)$.

(b) Suppose now $y(t) \uparrow \infty, t \geqslant t_{0}, t \rightarrow \infty$. If $\left|y^{\prime}(t)\right| \leqslant C_{0}<\infty$ then $|y(t)|^{\prime}<C_{0}$. Hence $y(t)=O(t)$ and the theorem is proved. Assume therefore that there is a sequence $t_{p} \uparrow \infty$ with $y^{\prime}\left(t_{p}\right) \uparrow \infty$. From (19) we have:

$$
\sum_{k_{0}=0}^{m_{0}} \frac{F_{0 k_{0}}(x)}{V^{m_{0}+k_{0}}(x)} y^{\prime m_{0}-k_{0}}=-\sum_{j=1}^{m} \frac{1}{y^{j}} \sum_{k_{j}=0}^{m_{j}} \frac{F_{j k_{j}}(x)}{V^{m_{0}-m_{j}+k_{j}}} y^{\prime m_{j}-k_{j}}
$$

and for $t=t_{p}$ with $p$ large enough one has

$$
\left|y^{\prime}\left(t_{p}\right)\right|^{m_{0}}-\sum_{j=1}^{m_{0}}\left|y^{\prime m_{0}-j}\left(t_{p}\right)\right| \leqslant \frac{1}{|y(t)|}\left|y^{\prime m_{0}}\left(t_{p}\right)\right| \Rightarrow(1+o(1))\left|y^{\prime m_{0}}\left(t_{p}\right)\right|<0 .
$$

But this inequality is impossible. Consequently $y^{\prime}(t) \leqslant C$.

(c) If $y(t) \downarrow-\infty$ then by means of the transformation $y=-z$ we reduce this case to case (b), which completes the proof of Theorem 2. 


\section{REFERENCES}

1. S. Bank, A general theorem concerning the growth of solutions of first-order algebraic differential equations, Compositio Math. 25 (1972), 61-70.

2. Amer. Math. Soc. 71 (1978), 39-45.

3.

, On the growth of solutions of algebraic differential equations, Trans. Amer. Math. Soc. 240 (1978), 195-212.

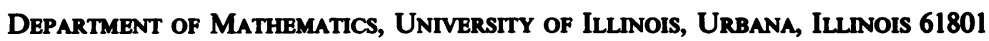

Current address: Department of Mathematics, University of Haifa, Haifa, Israel 